\title{
Recent Studies on Fundamentals and Application of Microwave Processing of Materials
}

\author{
Noboru Yoshikawa \\ Graduate School of Environmental Studies, \\ Department of Materials Science and Engineering, Tohoku University \\ 6-6-02, aza-Aoba, Aramaki, Aoba-ku,Sendai \\ ctopan
}

\section{Introduction}

Microwave (MW) application for heating was discovered in 1946 and has been applied in various fields. The detailed historical tracing is presented in the next section. Among the various aplications, it is possible to classify them into two major classes (Agrawal, 2005). In the first class, MW heating applied to drying, cooking foods and to excitation of chemical reactions such as inorganic/organic synthesis. The MW activated chemical reactions has been investigated in the field categorized as "MW Chemistry". In these applications, the heating temperature is usually low, because most of the aqueous and organic liquids have the boiling point below $500^{\circ} \mathrm{C}$. Mainly, the heating is caused by a dielectric relaxation loss due to rotation of molecules, which is as the result of the interaction of the MW electric field with the electric dipole of the molecules. Electric dipoles in liquid or gas experience relatively free rotational motion, comparing with the dipoles in a solid.

On the other hand, for the MW heating application to sintering of metal/ceramics, solid state reaction, solid state phase transition (such as vitrification or devitrification) and high temperature reduction reaction, elevated temperature is needed, which often exceeds above $500^{\circ} \mathrm{C}$. In these cases, heating mechanism is not limited to dielectric loss but to the other mechanisms of ohmic loss due to eddy current, and the magnetic loss also becomes important. Most of the oxides become electric conductive above $1000^{\circ} \mathrm{C}$ (Kingery, et al., 1975. From these considerations, understanding the effect of MW magnetic field interaction with materials becomes important (Roy, et al. 2002, Yoshikawa et al. 2006). As will be given in this paper, the magnetic field not only influences the magnetic heating mechanism directly, but also raises the induction current more effectively than in the electric field.

As the special characteristics of MW heating, three different heating aspects have been pointed out, namely they are the internal heating, the rapid heating and the selective heating (Bykov, et al., 2001). It is possible to consider the industrial application of MW heating from these aspects. Moreover, it has been reported that the so-called "non-thermal effect" of MW heating exists and enhances the sintering and the reaction kinetics. Although the origin of the non-thermal effect has not necessarily been clarified in the present stage, its phenomena keep providing us motivation to the reseraches on understanding and application of the MW heating. 
This INTEK book chapter is intended to introduce recent researches perfomed in the authors' group especially by virture of the project supported by Japanese Ministry of Education, Sports and Culture (MEXT), to be mentioned later. This paper starts with the historical tracing of MW heating researches. And it is aimed at concentrating the following viewpoints as the new microwave technologies:

1. Fundamentals in MW heating of materials in consideration of separated heating mechanisms in $\mathrm{E}$ - and $\mathrm{H}$ - field and the static $\mathrm{H}$ - field imposition.

2. Fundamentals and smart application of thermal runaway phenomena

3. Realization, interpretation and application of the long debating subject of MW nonthermal effects.

Concerning the above issues, our attempts of MW heating application have been conducted for the materials processing and the environmental technologies, which are simultaneously performed with the fundamental studies on the MW heating mechanisms together with some simulation studies.

\section{Historical}

Microwave (MW) has been utilized for tele-communication before and during the World War II, such as radar, sensing and so on. In 1946, Percy Spencer found a candy bar in his pocket was melting during his experiments on the MW generation tube, this is the discovery of MW heating (URL, 2010). Since then, MW heating has been applied to various fields (Clark, Sutton, 1996, Katz, 1992). At first, it was used in food production areas, such as drying of potato, roasting of coffee beans. It was in '70s, when the MW oven has become popular in domestic kitchens for cooking, as mass production of magnetron became posible at this time. And it was also in '70s when the oil shock or natural gas crisis occurred, which promoted the researches on MW heating in the western countries, because of their political necessity to be dependent on electric heating methods (Katz, 1992, Oda. 1992)

Application of MW heating to the energy and the environmental fields has also been attempted and realized. Their application extends to broad areas (Oda, 1992), such as incineration of medical wastes, devulcanization of rubber tire, treatment of sewage sludges, regeneration of spent activated carbon and chemical residues of petrol industries. In the nuclear engineering, MW de-nitration for producing MOX (Mixed OXide) nuclear fuels by re-disposal of the used plutonium (Kato et al. 2005). Vitrification of nuclear wastes by MW heating had been proposed in '80 (Morita et al. 1992).

In the area of materials processing, MW application to polymer has started earlier. There are bunch of research reports, curing of thermosets (Boey et al. 1992) is one of the examples. During $\mathrm{MW}$ drying of $\mathrm{Al}_{2} \mathrm{O}_{3}$ castable, it was recognized that not only well dried but also they can be heated well by MW (Sutton et al., 1988). MW heating of the ceramic powders above $1400^{\circ} \mathrm{C}$ made it possible to sinter ceramics (Janney and Kimrey, 1988). Later, it was reported that MW sintering enhances the diffusion rate, and so-called non-thermal effect (Wroe and Rowley, 1996) has been pointed out, though its origin has been a long debate. In this field, milli-wave techniques have been developed and applied to new processing of ceramics (Clark, Sutton, 1996).

On the other hand, metal heating was a minor application area of mirowave. And thus, the studies on MW heating of metals had not been developed comparing with the other materials as mentioned above. It is known that a bulk metal reflects MW, however, the metal particles and films can be heated well. And it is also known that ferro-magnetic metals 
can be heated more (Walkievicz et al., 1988), indicating that the magnetism is related with the heating mechanism.

Microwave metal heating researches are reviewed next. In 1988, one of an interesting experimental result is reported by Walkievics (Walkievicz et al., 1988), who tested heating of various metal powders, and demonstrated there are differences in their heating rates. In 1991-92, some reports related with heating of metals (cermets, composites) were presented in MRS symposium (Lorenson et al., 1991, Besher 1992). In '95, Mingos attempted synthesis of metal sulfide by MW heating of metals (Whittaker and Mingos, 1995). In '99, Roy et al. (Roy et al., 1999) reported microwave full sintering of metals in Nature magazine. And later, they attempted series of studies on heating of metals in the separated Electric (E-) and magnetic (H-) MW fields (Chen et al., 2002, Roy et al. 2002). In Europe, metal heating studies also have been performed (Rodiger et al.1998, Leonelli et al. 2008). These reports and activities motivated the MW researchers to be further directed to the metal heating studies. In Japan, the authors held a special symposium on metal heating in annual meeting of Japan Institute of Metals in 2005. The author also published a review article (Yoshikawa 2009(a)) in Bulletin of Japan Institute of Metals (Materia, Japan 2009, (written in Japanese)), the application fields are classified and the authors' recent results were introduced. Some content will be also presented in this article. The separated $\mathrm{E}$ - and $\mathrm{H}$ - heating (though not only metal heating) are directly related with the basic principles of microwave interaction with materials and of the microwave heating mechanisms.

In 2006, a project called "Grant in aid for priority field area" under support of Japanese Ministry of Education and Science was adopted and the intensive research activities are being conducted under the title of "Science and Technology of Microwave Induced, Thermally Non-Equilibrium Reaction Field". The author is one of the group leaders and promotes the area of "Microwave Application to the Mateials' Processing and Environmental Technologies".

Along with these activities, Japan Institute for ElectroMagnetic Energy Application (JEMEA) was established in 2006 and held a congress in 2008 together with the other international institutes of AMPERE, IMPI, MWG.

\section{Fundamentals}

he energy of electromagnetic wave is expessed in terms of Poynting vector $(\mathbf{E} \times \mathbf{H})$. Taking its divergence, and substituting the Maxwell's equations Eq. 1, 2 and 3. Eq. 4 is rearranged into Eq. 5, where E, H, B, D, Jare elctric and magnetic field, magnetic flux density, electric flux density and conduction current, respectively. and $\mathbf{B}, \mathbf{D}, \mathbf{J}$ are related with $\mathbf{E}$ and $\mathbf{H}$ according to Eq. 3, where $\varepsilon, \mu$ and $\sigma$ are permittivity, magnetic permeability and electric conductivity, respectively.

$$
\begin{gathered}
\nabla \times \mathbf{E}=-\frac{\partial \mathbf{B}}{\partial \mathbf{t}} \\
\nabla \times \mathbf{H}=\mathbf{J}+\frac{\partial \mathbf{D}}{\partial \mathrm{t}} \\
\mathbf{D}=\varepsilon \mathbf{E}, \quad \mathbf{B}=\mu \mathbf{H}, \quad \mathbf{J}=\sigma \mathbf{E}
\end{gathered}
$$




$$
\begin{gathered}
\nabla(\mathbf{E} \times \mathbf{H})=\mathbf{H} \cdot(\nabla \times \mathbf{E})-\mathbf{E} \cdot(\nabla \times \mathbf{H}) \\
=-\frac{\partial}{\partial t}\left(\frac{\varepsilon \mathbf{E}^{2}}{2}+\frac{\mu \mathbf{H}^{2}}{2}\right)-\sigma \mathbf{E}^{2}
\end{gathered}
$$

Eq.5 indicates that electromagnetic energy is stored in the matters as the first (electric field energy) and second (magnetic energy) terms. And the work done by the heating due to electric conduction current is expressed as the third term. The energy loss in the matters is evaluated by the first and the second terms using the imaginary part of the permittivity and the magnetic permeability, respectively. The Joule heating (induction heating) loss is expressed by the third term. They are corresponding to the dielectric, magnetic and conduction loss of the microwave heating.

The Maxwell equations (Eq.1,2,3) are converted to the exactly same differential equations with respect to the E- and H-fields for propagation of an EM wave, as follows:

$$
\nabla^{2}\left(\begin{array}{l}
\mathbf{H} \\
\mathbf{E}
\end{array}\right)-\mu \sigma \frac{\partial\left(\begin{array}{l}
\mathbf{H} \\
\mathbf{E}
\end{array}\right)}{\partial \mathrm{t}}-\mu \varepsilon \frac{\partial^{2}\left(\begin{array}{l}
\mathbf{H} \\
\mathbf{E}
\end{array}\right)}{\partial \mathrm{t}^{2}}=0
$$

In solving the equation, the time dependence of $\mathbf{H}$ (or $\mathbf{E}$ ) is assumed using exponential form, as Eq.7,

$$
\left(\begin{array}{l}
\mathbf{H} \\
\mathbf{E}
\end{array}\right)=\left(\begin{array}{l}
\mathbf{H}^{\prime} \\
\mathbf{E}^{\prime}
\end{array}\right) e^{-i \omega t}
$$

Then, the differential equation Eq.(6) has the same solution with respect to $\mathbf{H}$ and $\mathbf{E}$ as shown in one dimensional form in Eq.(8).

$$
\left(\begin{array}{l}
H^{\prime} \\
E^{\prime}
\end{array}\right)=\left(\begin{array}{l}
H_{0}^{\prime} \\
E_{0}^{\prime}
\end{array}\right) e^{-\alpha \cdot z} \cdot e^{-i(\omega \cdot t-\beta \cdot z)}
$$

Here, $\alpha$ is a characteriastic length of $H$ (or E) field attenuation, and $1 / \alpha$ is the length of the skin depth layer (d). $\alpha$ is expressed in Eq.(9).

$$
\alpha=\frac{\omega \sqrt{\mu \varepsilon}}{\sqrt{2}}\left[\sqrt{1+\left(\frac{\sigma}{\omega \varepsilon}\right)^{2}}-1\right]^{1 / 2}
$$

The EM field in metal must also obey Eq.(5), so $\alpha$ is also evaluated as Eq.(9). This indicates not only H-field but also E-field exists within the skin layer having the same thickness. In this treatment, generally, a relation $\sigma>>\omega \varepsilon$ is assumed for the metal case (Kraus ans Carver, 1973). Therefore $\varepsilon$ is canceled in Eq.(9) and a can be expressed as Eq.(10).

$$
\alpha=\sqrt{\frac{\omega \mu \sigma}{2}}
$$


And for the dielectric materials, Eq.9 is expresed, using a relation $\sigma=\omega \varepsilon^{\prime \prime}$

$$
\alpha=\omega \sqrt{\left(\frac{\mu_{0} \varepsilon^{\prime}}{2}\right)\left(\sqrt{1+\left(\frac{\varepsilon^{\prime}}{\varepsilon^{\prime \prime}}\right)^{2}}-1\right)}
$$

\subsection{Dielectric heating}

In considering the MW heating, it is required to estimate the penetration distance of MW into materials. The penetration distance of dielectrics depends mainly on the imaginary part $\left(\varepsilon^{\prime \prime}\right)$, so long as $\varepsilon^{\prime}>>\varepsilon^{\prime \prime}$ according to Eq. 9, this inequality relation holds in the most cases. On the other hand, the real part $\left(\varepsilon^{\prime}\right)$ influences the wave length within the matter. These relationships will be presented based on our recent studies.

In the case of dielectric heating, it is possible to estimate the penetration depth using Eq. 9 and the heat $(\mathrm{P})$ generated per unit time and unit volume, according to Eq. 12.

$$
P=\frac{1}{2} \omega \varepsilon^{\prime \prime}|E|^{2}
$$

In order to evaluate them, permittivity values $\left(\varepsilon^{\prime}\right.$ and $\left.\varepsilon^{\prime \prime}\right)$ are required to be measured at the MW frequency. In our group, the measurement was performed using cylindrical coaxial line method connected to vector network analyzer (VNA), as the schematic illustration of the apparatus shown in Fig. 1.
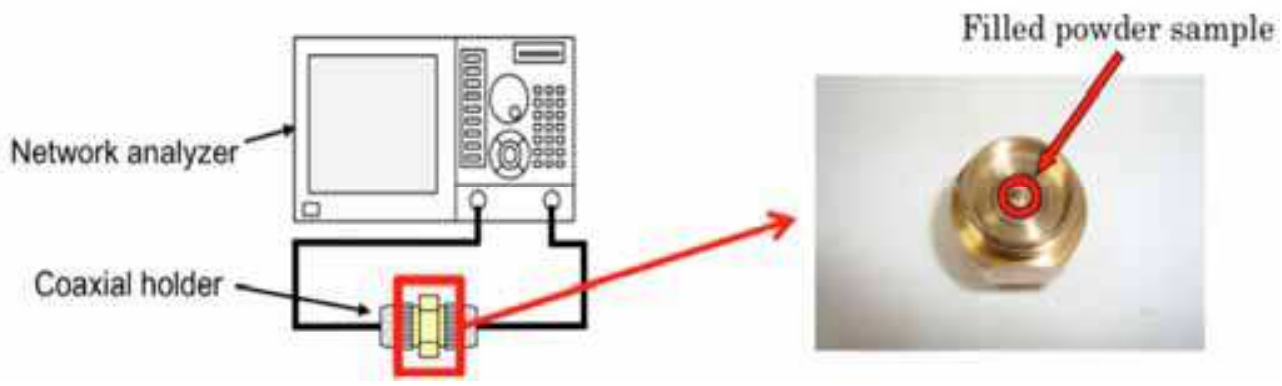

Fig. 1. Schematic illustration of apparatus for permittivity measurement.

Fist of all, the $\varepsilon^{\prime}$ effect on the wave length in a matter is demonstrated. In this case, not using the measured values by VNA but by the electromagnetic field simulation.

The simulation of the wave length of $\mathrm{MW}$ at $2.45 \mathrm{GHz}$ in a dielectric composite material with variation of $\varepsilon^{\prime}$ was performed. The composite material consists of spherical inclusion particles (perovskite) with large permittivity embedded in a matrix (spinel) with small permittivity. The inclusions were arranged in a configuration of face-centered cubic. The analyzed electric fields in the composite materials with inclusion having different particle diameters, corresponding to different average permittivity $\left(\varepsilon^{\prime}\right)$ are shown in Fig. 2 . The analysis demonstrates the local E-field distributions around the particles and the contours corresponding to the wave length. It can be seen that the wave length became shorter as an increase of average $\varepsilon^{\prime}$. 

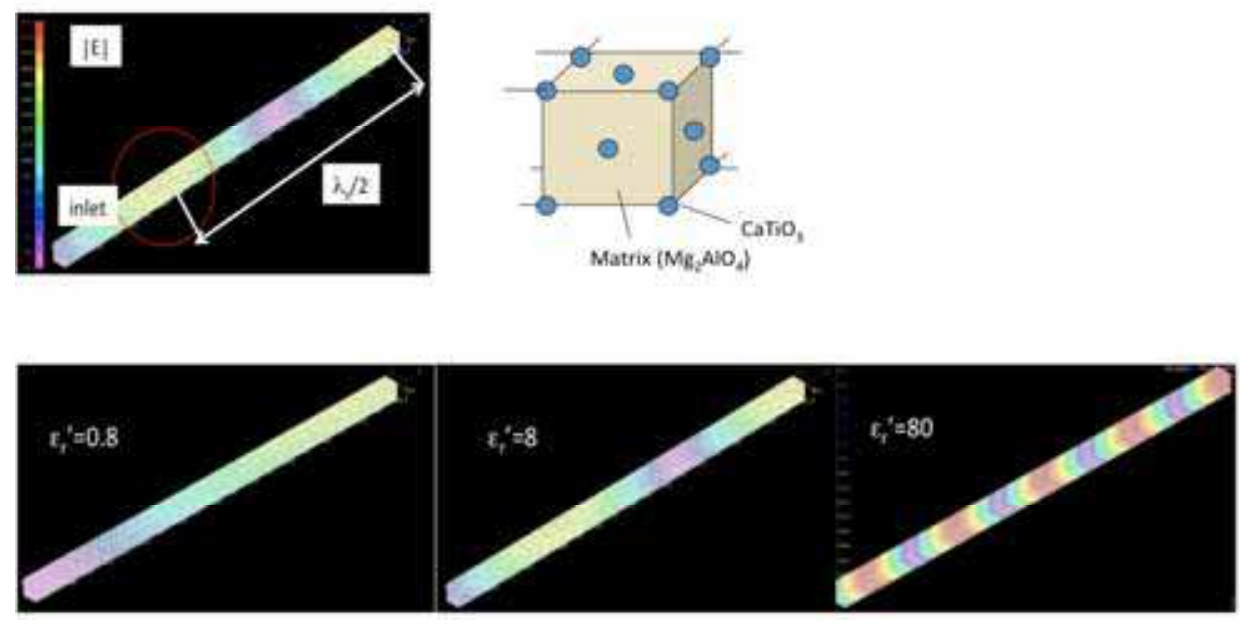

Fig. 2. Simulated electric field in a body consisting of 30 cells of ceramic composite. A cell shown upper right has dimension of $1 \mathrm{~mm}$ in cell length. The lower three images demonstrate the electric field distributions in the bodies consisting of inclusions with various size, corresponding to different average permittivity $\left(\varepsilon^{\prime}{ }^{\prime}\right)$.

The permittivity measurement was performed for the mixture of $\mathrm{FeOOH}$ and graphite powders. This mixture was selected as the test example for visualization of the microwave penetration distance, because $\mathrm{FeOOH}$ undergoes the following reaction by heating above $200^{\circ} \mathrm{C}$.

$$
2 \mathrm{FeOOH}=\mathrm{Fe}_{2} \mathrm{O}_{3}+\mathrm{H}_{2} \mathrm{O}
$$

The color changes from dark brown $(\mathrm{FeOOH})$ to brown red $\left(\mathrm{Fe}_{2} \mathrm{O}_{3}\right)$ upon heating. Here, $\mathrm{FeOOH}$ alone is not absorbing MW and not heated well, but graphite addition makes it possible (Iwasaki et al. 2009). It was observed that penetration distance decreased as the increase of graphite fraction and the degree of compression of the powder mixture. Photographs in Fig. 3 demonstrate the color changed areas where the MW penetration occurred and heating caused the above reaction. Decrease in the MW penetration distance at the larger graphite fraction is related with the permittivity $\left(\varepsilon^{\prime \prime}\right)$. The relationship is plotted in Fig. 4.

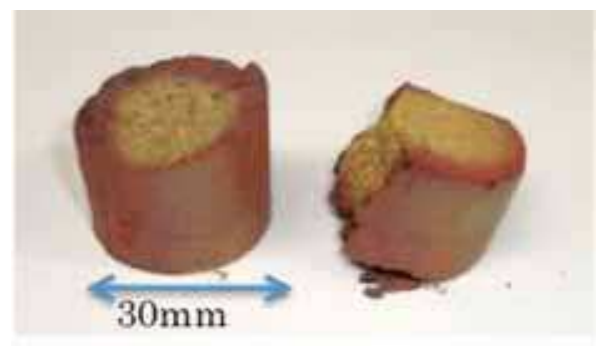

Fig. 3. Photo images of MW heated FeOOH/C mixture. 


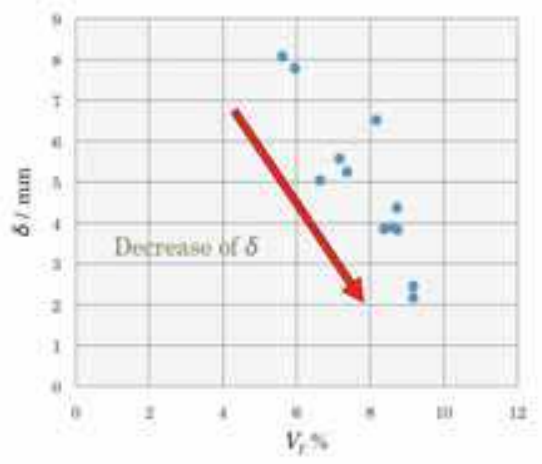

Fig. 4. Relationship between the $\mathrm{C}$ volume fraction and the estimated penetration distance.

\subsection{Induction (Metal) heating}

Because of small (order of micron or less) penetration depth of electromagnetic field into metals, temperature of a bulk metal cannot be raised very much, as mentioned above, therefore, MW heating is generally limited to metal particles or films. For example for $\mathrm{Au}$ case, according to Eq. 10, inputting $\sigma=4.6 \times 10^{7}[\mathrm{~S} / \mathrm{m}], \omega=2 \pi \times 2.45 \times 10^{9}[1 / \mathrm{s}], \mu_{\mathrm{r}}$ (relative magnetic permeability) $=1[-]$, the distance is estimated to be $1.5[\mu \mathrm{m}]$.

Ferro-magnetic metals are well heated by MW irradiation. Magnetic mechanisms are considered to be responsible, and our recent attempts to elucidating this mechanism will be presented in the next section (3.3).

Heating of non-ferromagnetic metals in electromagnetic field is primarily understood that the induced eddy current on the metal surface is responsible for the Joule heating. Or otherwise, occurrence of arcing accompanies the discharge current coming onto the metals surface, and also the Joule heating is brought about. The energy path generating the electric current and the current intensity are different between them. In this section, the eddy current (or induction current) shall be mainly concerned.

In discussion of the metal heating which is considered to occur only on the surface, it is required to discuss the electromagnetic boundary conditions on metal surface. Especially in this section it is intended to introduce the concept of surface impedance. MW heating of metals has to be analyzed with consideration of electromagnetic (EM) field above the metal surface, which must be linked with the EM field inside the metal surface or in the skin layer. It is usually understood that the MW H- field generates the induction (eddy) current, according to the boundary conditions of the electromagnetic field defined on the metal surface. The generally utilized boundary conditions on metal surface (so-called electric wall) assume the perfect conductor (or zero resistance), and is expressed as follows:

$$
\mathbf{E}_{1}=0, \quad \mathbf{n} \cdot\left(\mu \mathbf{H}_{1}\right)=0
$$

, where $\mathbf{n}$ is a normal vector to the metal surface. and

$$
\mathbf{n} \times \mathbf{H}_{1}=\mathbf{J}
$$

Jis a surface (eddy) current, as the schematic illustration shown in Fig. 5(a). Eq. 13 and 14 are the boundary conditions with respect to $\mathrm{E}$ and $\mathrm{H}$ fields in the air side on the metal 
surface, required for solving the EM fields in the given space (such as a wave guide and a cavity, etc). In this treatment, however, metal is idealized and no information about the metal properties is involved.

Induction (eddy) current is generated within the skin depth of the metal surface, which, of course, is due to the generated $\mathrm{E}$ - and $\mathrm{H}$ - fields in the metal because of the finite electric resistance. In order to describe the relationship between $\mathrm{H}$ - and E- field within the skin layer of metals, it is required to handle the boundary conditions of electric conducting (not perfect conductor, ie. having small but finite resistivity) metals. The analysis starting from Maxwell's equation is needed, which were already given above. And the penetration distance into metal is given in Eq. 10.

There are difficulties encountered in this analytical procedure. First, dielectric constant (permittivity: $\varepsilon$ ) of metal must be evaluated for the purpose of making comparison: $\sigma>>\omega \varepsilon$. Dielectric function of metals $\varepsilon(\omega)$ can be discussed using Drude model (Bohren and Huffman, 1983), however, this gives large negative value for the real part of $\varepsilon$ at MW frequency, which is confirmed by measurement of the optical constants (Johnson and Christy, 1972), however, this discussion is not usually performed in the textbooks of electromagnetics. Generally, in analysis of metals, displacement current $\frac{\partial \mathbf{D}}{\partial t}$ is neglected in Eq. 2 and thus Eq.6 can be rewritten as Eq. 15.

$$
\nabla^{2}\left(\begin{array}{l}
\mathbf{H} \\
\mathbf{E}
\end{array}\right)-\mu \sigma \frac{\partial\left(\begin{array}{l}
\mathbf{H} \\
\mathbf{E}
\end{array}\right)}{\partial \mathrm{t}}=0
$$

Permittivity measurement of metal powder has been performed and the measured values of $\varepsilon^{\prime}$ and $\varepsilon^{\prime \prime}$ (using an equation $\sigma=\omega \varepsilon^{\prime \prime}$ ) are incorporated into the EM field analysis and to the heating calculations (Mishra et al., 2006, Ma et al., 2007). However, the measured values are the 'effective' permittivity, and they could contain the influence of surface oxides and the air in the powder. The permittivity of metals or dielectric function to be used in solving the Maxwell equation must be related to the electronic states of metals.

Second, as there is an eddy current $\mathbf{J}$ generated by time derivative of $\mathbf{H}$. Here, if we relate $\mathbf{J}$ to $\mathbf{E}$ by Eq.15, this indicates the $\mathbf{E}$ field also exists in the skin layer, and there are relations among $\mathbf{J}, \mathbf{E}$ and $\mathbf{H}$. So, the boundary condition Eq.(1) has to be modified to express them. Landau and Lifshitz in their textbook (Landau et al., 1984) discussed this problem in terms of surface impedance of metals (originally by L.A.Leontovich, 1948). As can be derived from Eq. 9, both $\mathrm{E}$ and $\mathrm{H}$ fields exist within the surface skin layer $\delta$ as $\delta=1 / \alpha, \alpha$ is expressed by Eq. 10. On the other hand, the tangential $\mathrm{E}$ and $\mathrm{H}$ fields in metal surface is related by the following relation (t: tangential, 2: metal medium, as shown in Fig.5(b)) : $E_{2 t}=\zeta_{s} H_{2 t}$, where $\zeta_{\mathrm{s}}$ is a surface impedance expressed in cgs unit (in Landau and Lifshitz) by

$$
\varsigma_{s}=\sqrt{\frac{\omega \mu}{8 \pi \sigma}} \cdot(1-i), \text { or } \varsigma_{s}=\sqrt{\frac{\mu \omega}{2 \sigma}} \cdot(i-1) \quad \text { (in MKSA unit) }
$$

and its value has an order of $\sim \delta / \lambda$ ( $\lambda$ : wave length), indicating that $\mathrm{H}$ field is larger than $\mathrm{E}$ field on the metal surface at the microwave frequency $\left(\delta / \lambda \sim 1 \times 10^{-4}\right)$. This aspect is also demonstrated in the textbook of J.D.Jackson (Jackson, 1998). Using this quantity, the boundary condition Eq. 13,14 are modified to the below form: 


$$
\mathbf{n} \times \mathbf{E}_{1}=\zeta_{\mathrm{s}} \mathbf{n} \times\left(\mathbf{n} \times \mathbf{H}_{1}\right)
$$

This is the boundary condition of $\mathrm{E}$ and $\mathrm{H}$ fields of the space as well, which considers the metal properties. And again, larger contribution of $\mathrm{H}$-field to metal heating was confirmed.
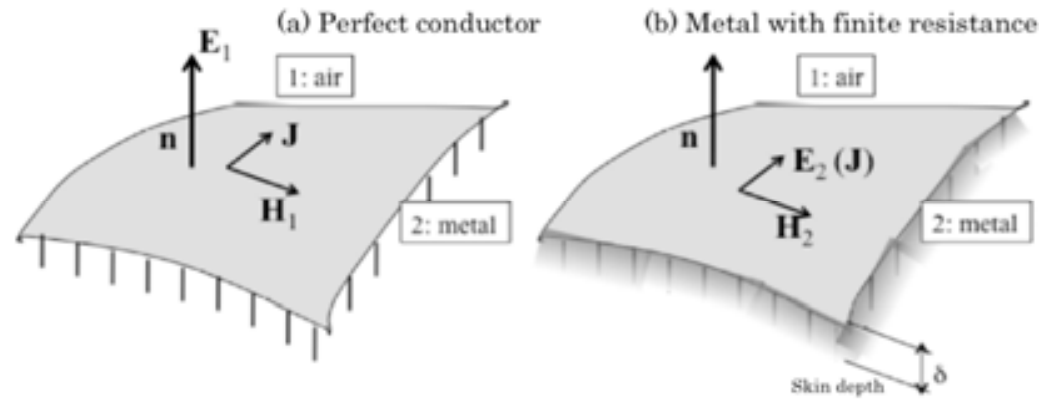

Fig. 5. Schematic illustration of EM boundary conditions on metal surface.

\subsection{Magnetic heating}

It is commonly recognized among the microwave processing researchers that generally the ferro (ferri)-magnetic materials are well heated by microwave, especially in H-field. And they can be heated better up to the Curie temperature (Ishizaki and Nagata, 2007). These facts indicate the magnetic mechanism plays an important role in microwave heating, however, this mechanism is not necessarily clarified enough, so far. On the other hand, in designing RF devices such as ferrite cores, the RF loss occurring in a range of $10^{8} \sim 10^{9} \mathrm{~Hz}$ is a detrimental phenomenon and is necessary to be avoided (Pardavi-Horvath,2000). This loss is especially termed as a natural resonance (Polder and Smit, 1953). The natural resonance is related with the internal magnetic fields existing within the ferro (ferri)-magnetic materials, by which the precession movement of electron spin occurs. As the precession frequency corresponds the ranges mentioned above, the resonant absorption of microwave takes place. In general, as there are relatively large distributions of the internal magnetic field, so the resonant frequency also has broad ranges.

Natural resonance takes place without external magnetic field. Here, it is expected possible to raise the electron spin resonance (FMR) in ferro (ferri)-magnetic materials by imposition of static external magnetic field. Namely, if the natural resonance is one of the mechanism responsible for microwave magnetic heating, it might be possible to expect the extra heat generation due FMR and to observe the temperature rise.

FMR has been studied extensively from ' $50 \mathrm{~s}$, and a bunch of reports on the experimental and theories are published. However, most of the studies were performed on the spin-spin relaxation or spin wave dynamics by the physics school (Sparks, 1964). Therefore, the specimen used was mainly an yttrium iron garnet (YIG) having longer spin-spin relaxation time, and not easy to be converted into heat. Thus far, less studies was performed on the application of FMR to heating materials, except a few reports (Nikawa and Okada, 1987 and Walton et al., 1996), but no intimate examination under various FMR conditions were provided. This study (Yoshikawa and Kato, 2010) attempted to observe the temperature change of $\mathrm{Fe}_{3} \mathrm{O}_{4}$ compressed body by setting the FMR conditions. And it is intended to investigate the FMR heating behavior dependence on various conditions, and then to discuss the magnetic energy dissipation to heat. 
$\mathrm{Fe}_{3} \mathrm{O}_{4}$ powder of $4 \mathrm{~N}$ in purity, which were compressed uniaxially and formed into a rod having $7 \mathrm{~mm}$ in diameter and $4 \sim 0.5 \mathrm{~mm}$ in thickness, the density of which are about $1 \times 10^{3} \mathrm{kgm}^{-3}$. The specimens were placed at the bottom of silica glass rod container. The specimen was placed in a $5.8 \mathrm{GHz}$ microwave TE103 cavity at the $\mathrm{H}$-field maximal position and the static magnetic field was imposed perpendicular to the microwave magnetic field.

The specimen temperature was measured by an optical method using a sapphire light guide. $\mathrm{N}_{2}$ gas was flown from downward onto the specimen surface and no heat insulating materials were set above the specimens.

The specimens were heated up to the pre-determined temperature in microwave $\mathrm{H}$-field without imposition of Hext. Data of the temperature variation with time were digitally recorded, together with that of $\mathrm{Hext}^{\mathrm{e}}$, the forward and backward reflection power of microwave (Pf and Pr, respectively).

Curves of the temperature variation with time obtained from $\mathrm{Fe}_{3} \mathrm{O}_{4}$ compressed body of $0.5 \mathrm{~mm}$ in thickness are obtained plotted in Fig. 6, together with Pf, $\mathrm{Pr}$ and $\mathrm{Hext}^{\mathrm{ex}} \mathrm{Fe}_{3} \mathrm{O}_{4}$ compact was preset at $420^{\circ} \mathrm{C}$ and kept for couple of ten seconds, $\mathrm{H}^{\mathrm{ext}}$ was imposed. It was demonstrated that the temperature increased and had a peak $(\Delta \mathrm{T}$ : the temperature difference from the initial preset value) was observed then decreased (a). The peak appeared at almost same $\mathrm{H}^{\mathrm{ext}}$ during ascending and descending of $\mathrm{H}^{\mathrm{ext}}$, as can be seen clearly by plotting the temperature with respect to $\mathrm{Hext}^{(b)}$ indicating that FMR induced the temperature rise $(\Delta \mathrm{T})$. It can be seen from the figure that Pr decreased a little around the Hext of the resonance. This is considered to be casused by the energy absorption by magnetic resonance. As mentioned above, $\Delta \mathrm{T}$ could become larger if heat insulation condition is provided and there is no gas blow.

The resonant $\mathrm{H}^{\mathrm{ext}}$ is supposed to be influenced by demagnetization field depending on the specimen shape. Namely, Kittel resonance (Kittel, 1948) condition has to be taken into consideration. Without any additional magnetic fields, such as anti-magnetic field, the resonance field is expected to be $0.21 \mathrm{~T}$ at $5.8 \mathrm{GHz}$. The resonant $\mathrm{Hext}$ of about $0.15 \mathrm{~T}$ is interpreted as follows: The compressed $\mathrm{Fe}_{3} \mathrm{O}_{4}$ powder is not a continuum body, and the resonant frequency is determined by each particle. The powder particles do not have complete spherical shape, and the demagnetization conditions are deviated from the sphere case (if complete sphere, $\mathrm{H}^{\mathrm{ext}}$ is not affected by the demagnetization field.).

In this study, dependence of $\Delta \mathrm{T}$ and the resonant $\mathrm{H}^{\mathrm{ext}}$ on the pre-heated temperature was examined and the obtained relationships are plotted in Fig. 7(a)(b). These data provide us information how the magnetic mechanism for microwave heating varies with the temperature. It was shown that $\Delta \mathrm{T}$ decreased as the pre-heated temperature approached to their Curie point. And the resonant $\mathrm{Hext}^{\mathrm{s}}$ shifted to higher. These phenomena are primarily interpreted as the decrease in the saturated magnetization $\left(I_{s}\right)$. The magnetic resonance in ferro-magnetic state may be able to give rise to the observable heat generation (temperature increase) and disappears as approaching to the paramagnetic state.

The FMR is a state that the unpaired spins contributing to ferro-magnetism are precessing altogether around the (external) magnetic field direction in resonant conditions with the applied microwave magnetic field. Namely, they correspond to the spin wave number $k=0$ (Sparks et al., 1961). The process of the resonant precessing energy converting to heat is different from the other heating mechanisms such as Joule heating of eddy current. The differences are either magnon-phonon interaction or that of electron-phonon. it is expected that FMR heating to become a promising heat treatment method of magnetic materials giving rise of excellent properties. 

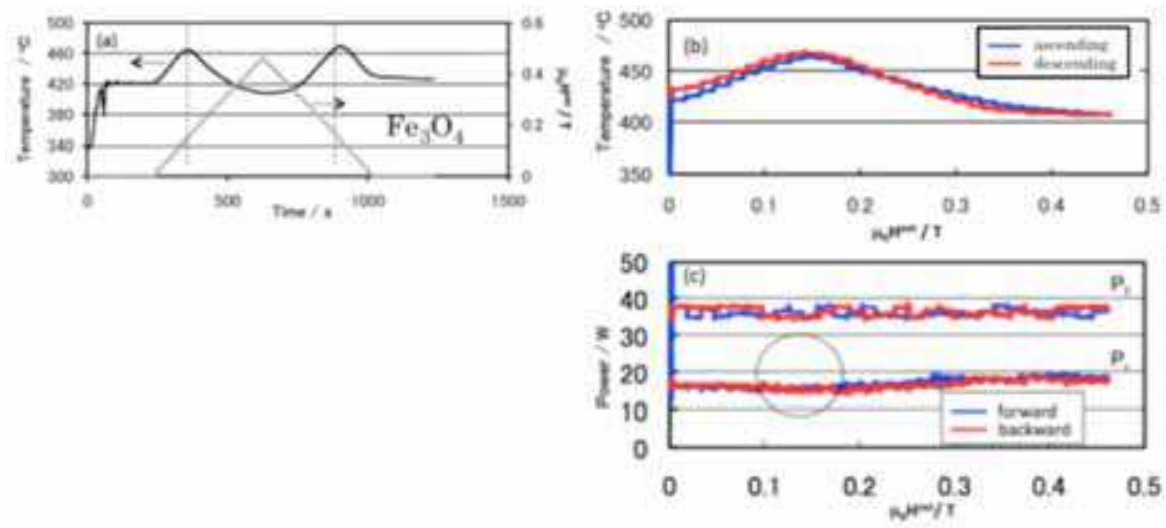

Fig. 6. Temperature of $\mathrm{Fe}_{3} \mathrm{O}_{4}$ rod and Pf,Pr variations during imposition of external magnetic field (Hext). (a) Variation of temperature and $\mathrm{H}^{\mathrm{ext}}$ with time. (b) Temperature plotted with respect to $\mathrm{Hext}^{\mathrm{e}}$.
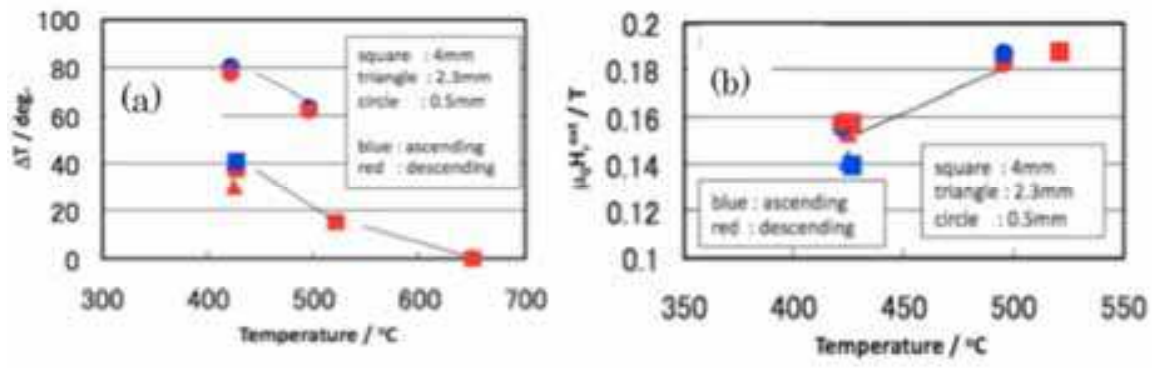

Fig. 7. (a) Temperature increase $(\Delta \mathrm{T})$ from the initial state plotted with respect to the preheated (initial) temperatures with different thickness. (b) Resonance external magnetic field $\left(\mathrm{H}_{\mathrm{r}}{ }^{\mathrm{ext}}\right)$ plotted with respect to the pre-heated temperatures. $\left(\mathrm{Fe}_{3} \mathrm{O}_{4}\right.$ compact) The blue and red marks represent the $\Delta \mathrm{T}, \mathrm{H}^{\mathrm{ext}}$ values obtained in ascending and descending of $\mathrm{H}^{\mathrm{ext}}$, respectively. Keys in (b) are square; $4 \mathrm{~mm}$, triangle; $2.3 \mathrm{~mm}$, circle; $0.5 \mathrm{~mm}$.

\section{Applications}

\subsection{E-H Separated heating of thin metal films}

Recently, separated E- and $\mathrm{H}$ - field heating has been performed for the purpose of investigating the heating mechanisms and of discriminating the heating behaviors to fabricate new materials. In these experiments, most commonly observed tendency is that $\mathrm{H}$ field is more effective for heating of metals comparing with the E- field, as first reported by Cheng et al. (Chen et al. 2001) and the authors have confirmed in series of experiments (Yoshikawa et al., 2006).

Heating behaviors of $\mathrm{Cu}$ particles by MW irradiation in the separated E- and H- fields was reported (Ma et al., 2007), where it is mentioned that heating rate in H-field is higher, and better heating behavior was observed in the subsequent heating. And it is to be noticed that the heating curve (time-temperature) exhibits peaks at the initial stage of heating. The 
authors of this report attributed this behavior to be due to the microstructural change such as the particle contacts formation and growth of average particle size, which occurs within the skin depth of MW penetration.

In our recent work on heating of Au films with nano-sized thickness, peak formation behavior was also observed, as shown in Fig. 8 (Cao et al., 2009). In this case, film thickness is less than that of the skin layer. Although there is a difference in materials, it is of interest to compare these behaviors with the $\mathrm{Cu}$ cases. It was also confirmed that $\mathrm{MW} \mathrm{H}$-field is effective for heating the films (same input power resulted in different temperature). The film grain structure was also altered by MW heating, as the XRD profiles indicated in Fig. 9. The changes in grain structure alter the MW absorbability and resulted in the peak formation. This must also be discussed in terms of the change in surface impedance, namely it is caused by some alteration in interaction between electromagnetic wave and metals. The reason for the better heating characteristics of metal particles and films in $\mathrm{H}$-field is also interpreted as the difference in the eddy current. Further detailed studies are expected to be explored in future. Apart from the fundamentals in the metal film heating, heating of metal thin film has been successfully applied to the crystallization of PZT film in the multi layered structure (Wang et al. 2008).

(a) H-field

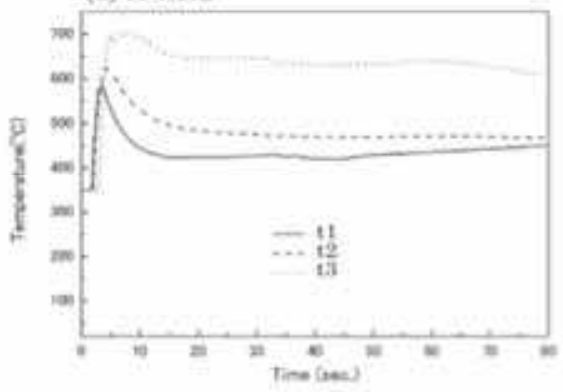

(b) E-ficld

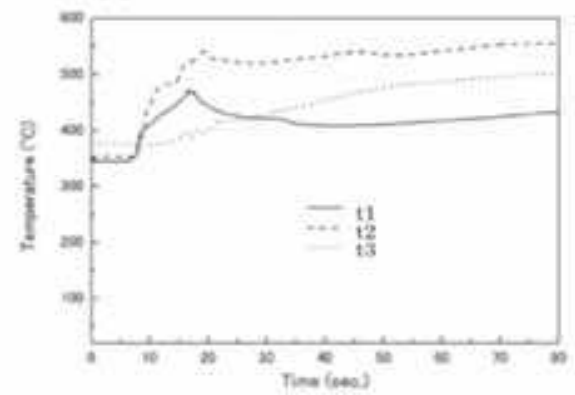

Fig. 8. Heating curves of Au films having various thickness (t1: 45nm, t2: 133nm, t3: 407nm) in H- and E-field (Cao et al., 2008).

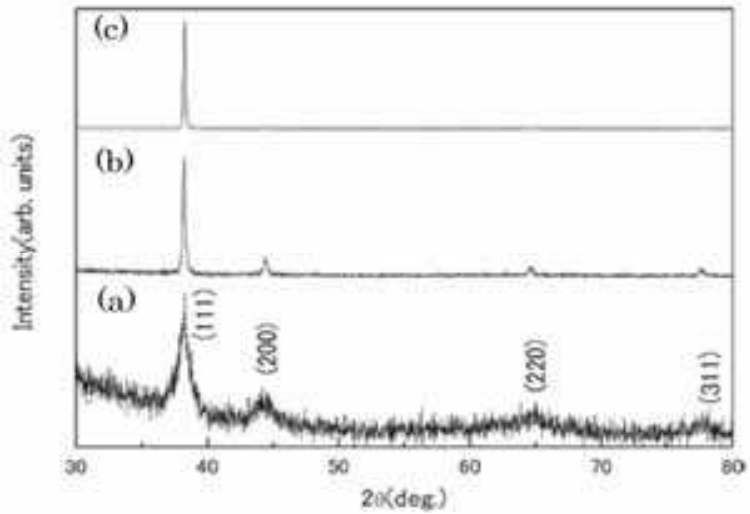

Fig. 9. XRD profiles of Au films (t2) of (a): as deposited, (b) heated in E-field and (c) heated in H-field (Cao et al., 2008). 


\subsection{Thermal runaway}

\subsubsection{MW heating behaviour of Fe/Soda-lime $\left(\mathrm{Na}_{2} \mathrm{O}-\mathrm{CaO}-\mathrm{SiO}_{2}\right)$ glass mixture}

For the purspose of glass solidification of hazardous wastes, metal pieces are mixed for promoting the MW heating. We fablicated the soda-lime (S-L) glass/metal composite materials (Yoshikawa et al., 2008(a)). It is of significance to investigate the MW heating of glass with additon of metals.

Heating behavior of spherical S-L glass beads having $0.2 \mathrm{~mm}$ in diameter was examined, first. The heating curves by MW irradiation of constant (maximum) power indicated that the attained maximum temperature was about $200^{\circ} \mathrm{C}$ after 10 minutes of MW irradiation (not shown here).

Iron powder was heated up to about $700^{\circ} \mathrm{C}$ rather quickly and saturated within $10 \mathrm{~min}$. This behavior was discussed in relation with the Curie point of iron $\left(770^{\circ} \mathrm{C}\right)$ (Yoshikawa et al., 2006), though the maximum temperature attained is influenced by the state of thermal insulation.

Next, mixture of S-L glass beads and iron powder was heated with various volume fraction of iron powder, as shown in Fig. 10. It was observed that the mixture of small volume fraction ( $2.2 \mathrm{vol} . \%)$ of iron powder has essentially same heating behavior as the glass beads alone. On the other hand, the temperature rose quickly within one minute in the mixture of $18 \mathrm{vol} \% \mathrm{Fe}$. There is a transition state in the volume fractions of iron, in which temperature rose up suddenly after certain incubation time periods. It can be seen that the temperature rise was so rapid and this might be a phenomenon so called thermal runaway (TRW) (Comm. on Microwave Proces. Mater., 1994, and Kenkre eat al., 1991). The MW power was shut off shortly after the onset of the sudden rise in order to protect the thermocouple. The incubation time decreased as an increase of iron volume fraction. The heated specimens were observed with $\mathrm{OM}$, and the microstructures are illustrated in Fig. 11. It can be seen that the iron powder fills the clearance of the beads spheres (at $800^{\circ} \mathrm{C}$ ), and the glass beads are melted at $950{ }^{\circ} \mathrm{C}$. The iron powder was neither melted in this temperature nor reacted with the S-L glass, which was examined with SEM/EDX observation.

Pure silica glass $\left(\mathrm{SiO}_{2}\right)$ is not a good MW absorber, however the permittivity ( $\varepsilon^{\prime \prime}$ : loss factor) increases as the temperature, because of increase in population of mobile ion or ion defects (Kenkre et al. 1991), resulting in interaction with the MW electric field. It is also known (Kingery at al., 1975) that this phenomenon is related with the large increase in electric

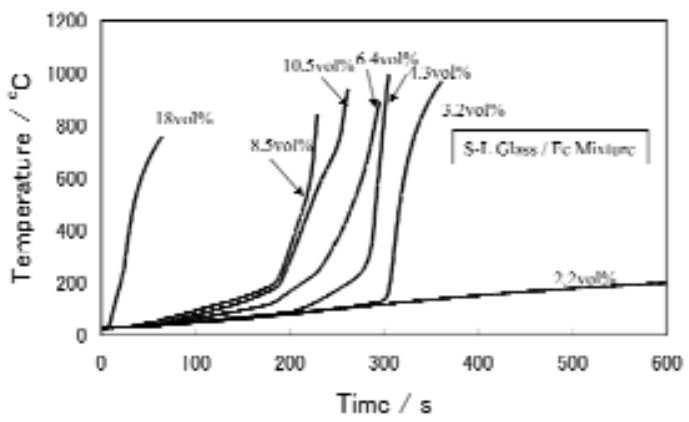

Fig. 10. Heating curves of S-L glass/iron powder mixture with different volume fraction of iron powder. 


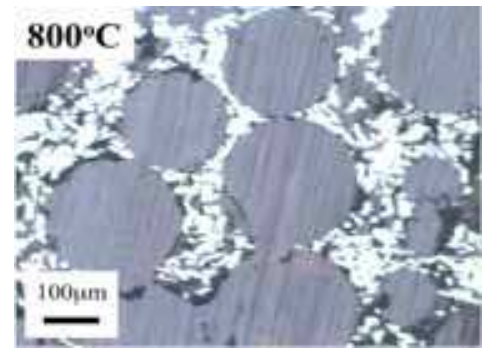

Fig. 11. Optical micrographs of MW-heated S-L glass/iron powderheated at $800^{\circ} \mathrm{C}$ with $18 \mathrm{vol} \% \mathrm{Fe}$.
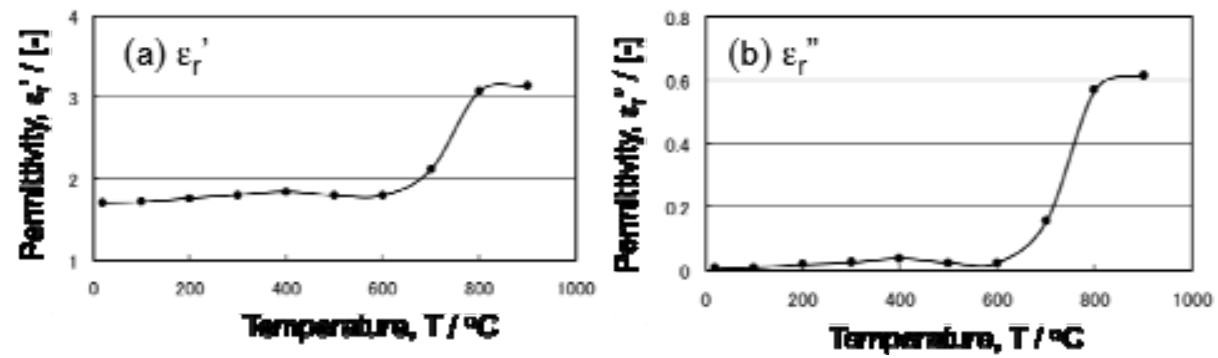

Fig. 12. Temperature dependence of (a) real $\left(\varepsilon_{\mathrm{r}}{ }^{\prime}\right)$ and $(\mathrm{b})$ imaginary $\left(\varepsilon_{\mathrm{r}}{ }^{\prime \prime}\right)$ parts of S-L glass permittivity.

conductivity at high temperature as mentioned. In this study, the permittivity of S-L glass was measured up to the elevated temperature (Yoshikawa et al., 2009(b)), and plotted in Fig. 12. As can be seen from the plot, permittivity (both $\varepsilon^{\prime}$ and $\varepsilon^{\prime \prime}$ ) increases mainly above $700^{\circ} \mathrm{C}$. Therefore, plausibility of the hypothesis was confirmed, and it became possible to discuss the heating behavior of the mixture of S-L glass and metal powders.

The S-L glasses were not heated well above $200^{\circ} \mathrm{C}$ within 10 minutes in a multimode applicator without addition of iron powder, probably because the loss factor of the glass below $200^{\circ} \mathrm{C}$ was not large enough to cause the heating. Iron powder is heated well up to about $700^{\circ} \mathrm{C}$. This makes it possible to heat the S-L glass beads in the neighbors of metal powder, and increased the loss factor of the glass, leading to the further heating of the glass beads by themselves (glass $+10 \mathrm{vol} \% \mathrm{Fe}$ in Fig. 10). The S-L glass might be heated by MW Efield component, mainly by dielectric heating mechanism (though interaction of the enhanced electric conductivity with the $\mathrm{H}$-field is also of interest.).

Occurrence of thermal runaway (TRW) was dependent on the volume fraction of iron powder. It is of interest how the volume fraction of Fe powder determines the onset of sudden temperature rise.

Although MW penetration depth into metal is of an order of microns or less, so the heat is generated only in this surface skin layer. Heat conduction within the small metal particle is so quick, and thus the particles might be heated quickly. Therefore, it is possible to consider that the aggregates of iron powder are taken to be internally heated by MW, if we assume sufficient penetration of MW into the aggregates. In other words, the aggregates are considered to be a volumetrically heated objects by MW. The glass beads are heated by these metal aggregates. This feature is schematically illustrated in Fig. 13. As an increase of 
the metal powder fraction, the clearance of glass beads is filled with the larger aggregates of metals particles. If so, they can be heated more than the small aggregates, because the larger volume generates the larger heat with respect to the smaller heat transfer through the surface area. (The heat generated in the aggregate is propotional to the cube of its radius, while the heat transfered from the aggreagte is propotional to the square of ist radius. Therefore, temperature rises the higher as an aggregate has the larger size.)

Occurrence of TRW must be determined due to superposition of the following phenomena: One is the above discussed temperature dependence of the S-L glass permittivity and the other is the size dependence of the heat generated by the metal powder aggregates.

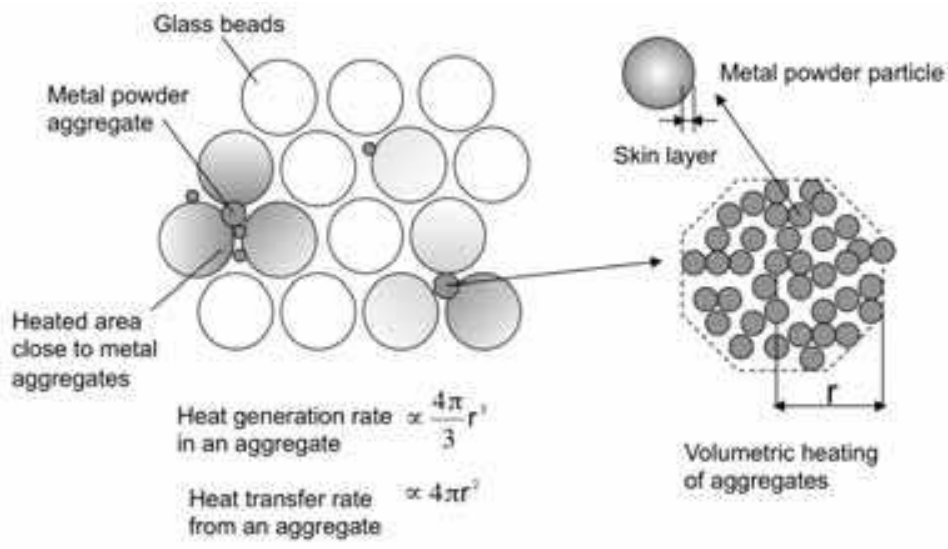

Fig. 13. Schematic illustration of S-L glass beads heating with an aid of metal aggregates.

\subsubsection{MW heating for carbo-thermal reduction of $\mathrm{Cr}_{2} \mathrm{O}_{3}$ (Yoshikawa et al. 2008(b))}

In order to recycle $\mathrm{Cr}$ metals from the wates occurring in stainless steel production process, such as the pulverized Cr-containing slags and picking sludge, they are reduced with graphite via carbo-thermal reduction reaction. Owing to the large affinity of chromium with oxygen, chromium oxide $\left(\mathrm{Cr}_{2} \mathrm{O}_{3}\right)$ reqires high reduction temperature as predicted by thermodynamics. However, because of the specific dependence of MW absorbability of $\mathrm{Cr}_{2} \mathrm{O}_{3}$, it is possible to reduce the (average) reduction temperature. First of all, the reduction of real wastes are presented.

Without graphite addition, the real sludge and the Cr-containing slag powder were not heated by MW above $200^{\circ} \mathrm{C}$. This is because of the fact that fractions of heavy metal oxides are lower with respect to the gangue components such as $\mathrm{CaO}, \mathrm{SiO}_{2}$, and the silicates. Mixture of either slag or sludge with sufficient amount of graphite was successfully heated by $\mathrm{MW}$ and the reduced $\mathrm{Cr}$-containing alloys were obtained.

SEM/EDX photographs of the reduced specimens are shown in Fig. 14. The Fig. 14(a) illustrates the $\mathrm{Cr}$-containing slag specimen heated at $1000^{\circ} \mathrm{C}$ for $10 \mathrm{~min}$., with graphite addition of $5 \mathrm{wt} \%$. The bright area corresponds to the reduced metal (alloy). Composition of the reduced alloy was determined. The obtained $\mathrm{Cr}$ concentration values differed from the different areas, probably because of the original segregation of $\mathrm{Cr}$ in the oxide states.

MW heating behavior of $\mathrm{Cr}_{2} \mathrm{O}_{3}$ was investigated by measuring its heating curves (timetemperature relationship) in a multi-mode applicator and compared with that of graphite 
and $\mathrm{NiO}$. They were measured under the same input power of $670 \mathrm{~W}$ (maximum), and the plots are shown in Fig. 15.

Graphite can be heated very well, and temperature rose up to $1000^{\circ} \mathrm{C}$ within $2 \mathrm{~min}$. On the other hand, $\mathrm{NiO}$ and $\mathrm{Cr}_{2} \mathrm{O}_{3}$ had incubation time before onset of the rapid temperature increase. Although the incubation time periods of $\mathrm{Cr}_{2} \mathrm{O}_{3}$ is similar to $\mathrm{NiO}$, temperature of $\mathrm{NiO}$ saturated at $900^{\circ} \mathrm{C}$, but temperature of $\mathrm{Cr}_{2} \mathrm{O}_{3}$ still increased. In this case, power was switched off in order to avoid damaging the thermo-couple.

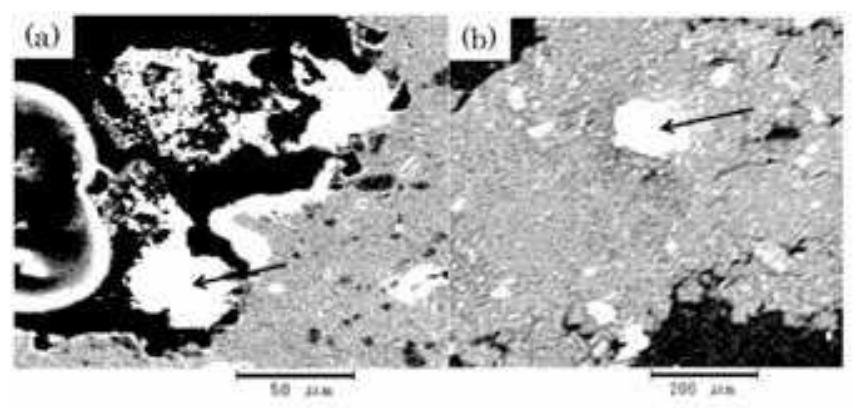

Fig. 14. SEM images of MW-reduced alloys from (a) Cr-containing steel making slag and (b) stainless steel pickling sludge. The bright areas indicated by arrows are the reduced alloys, the EDX-analyzed compositions (at\%) are (a) Fe 88.5(bal.), Cr 11.5 and (b) Fe 58.3(bal.), Cr 7.3, Ni 33.9

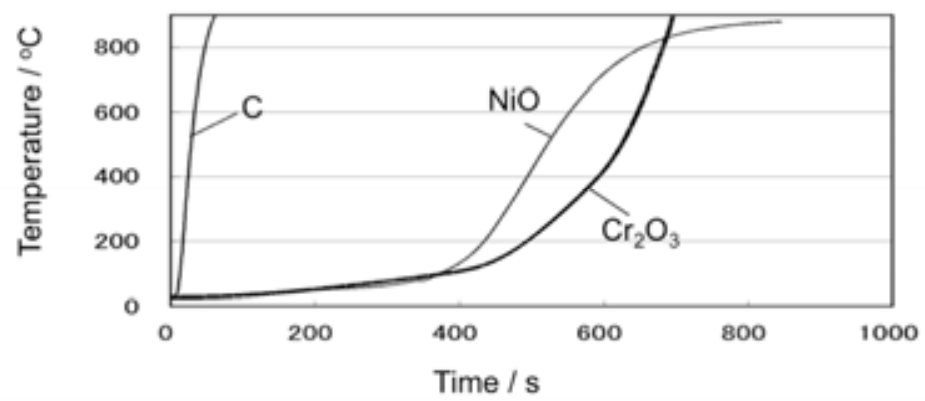

Fig. 15. Heating curves of graphite (C), $\mathrm{NiO}$ and $\mathrm{Cr}_{2} \mathrm{O}_{3}$ powders in a multi-mode applicator at maximum power $(670 \mathrm{~W})$.

Next, $\mathrm{Cr}_{2} \mathrm{O}_{3}$ powders were heated in a single-mode MW applicator. Separated E- and $\mathrm{H}$ field heating provides information to discuss the MW heating mechanisms. The heating curves are plotted in Fig. 16 ( $\mathrm{NiO}$ heating curve in E- and $\mathrm{H}$-field (Yoshikawa, 2007) Although there was an incubation time before onset of the temperature rise, E- field MW irradiation enabled high temperature heating, while $\mathrm{H}$ - field application was not successful to heat it above the detection limit of the pyrometer (saphire light pipe). These facts show that the dielectric heating mechanism is important in heating of $\mathrm{Cr}_{2} \mathrm{O}_{3}$, other than the magnetic and the ohmic loss mechanisms.

Not only the Cr-containing oxides' waste but also pure $\mathrm{Cr}_{2} \mathrm{O}_{3}$ were reduced at lower temperature than that expected from thermodynamics. In these experimental settings, the 
temperature is measured from the localized area in the specimen. Namely, some finite area on the surface temperature is measurable by an optical method $(2 \mathrm{~mm}$ in the optical rod diameter), and a thermocouple sheath in the inside of the powder (having diameter of $3 \mathrm{~mm})$. This means there could be some areas having much higher temperatures locally, as schematically illustrated in Fig. 17. In these considerations, it is expected that the reduction occurred at the high temperature regions where the thermodynamic conditions are satisfied. It is plausible to assume existence of the local temperature fluctuation at the initial stage of the MW heating, for example, because of the $\mathrm{Cr}_{2} \mathrm{O}_{3}$ and graphite grain size distribution or their aggregates' size in the mixture according to the mixing conditions, although the degree of their difference is not clear. If there are regions having differnces in local temperature, it is inferred that the high temperature region can be heated preferentially, because of the permittivity increase, as the case of S-L glass shown in 4-2.1, and thus the temperature fluctuations are expected to be amplified. However, other possibility of arcing effects cannot be still discarded.
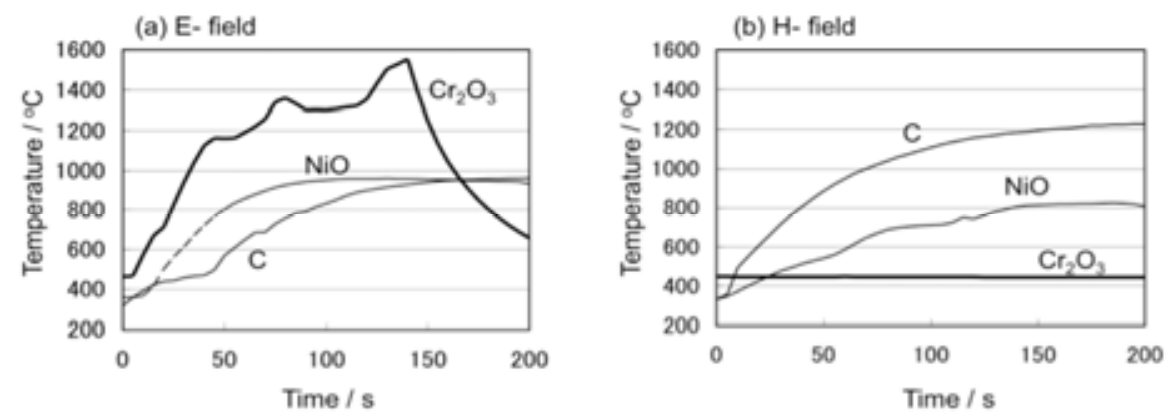

Fig. 16. Heating curves of graphite (C), $\mathrm{NiO}$ and $\mathrm{Cr}_{2} \mathrm{O}_{3}$ powders in a single-mode applicator at input power of 200W, (a) in E-field and (b) in H-field.
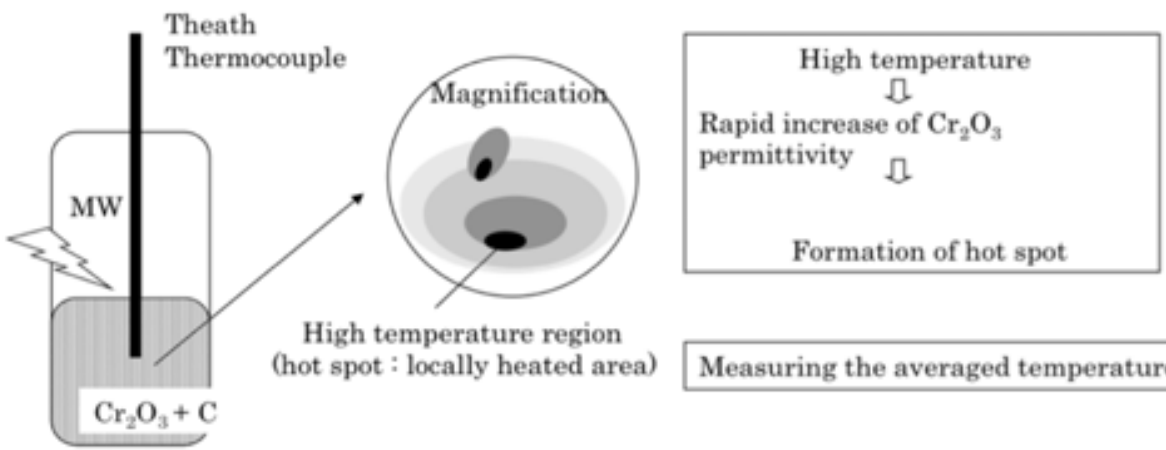

Measuring the averaged temperature

Fig. 17. Schematic illustration of models of the enhanced reaction kinetics and formation of metal pieces.

Accordingly, it is possible to cause reactions using TRW at the average temperature lower than that expected from thermodynamics. And the reaction process could be finished within the shorter time period. Therefore, it is of significance to take advantage of this phenomenon 
for the practical application. However, it is important to note that the controllability of the TRW process is not necessarily easy.

\section{Discussion on the non-thermal effects}

\section{Classification of the phenomena}

There have been many reports concerning on the so-called non-thermal effect in MW heating. It would be of significance to describe the interpretation and possibilities for realization of the non-thermal effects in discussion of the our results presented in this paper. The arguments are to be divided into three classes.

\subsection{Case 1: The thermal effects appear to be non-thermal}

Hot spot is sometimes brought about because of selective heating. Starting with the fluctuation of the temperature, rapid increase in temperature occurs locally due to the large temperature dependence of permittivity (loss factor) at high temperature. The examples are presented in 4.2 for the cases of $\mathrm{S}-\mathrm{L}$ glass and $\mathrm{Cr}_{2} \mathrm{O}_{3}$. It was mentioned however, that taking advantage of the rapid increase of temperature or TRW is one of the new points of view for the application of MW heating.

On the other hand, it has to be noted that the temperature measurement performed in the presence of hot spot or temperature distribution provides the erroneous information. For examle, thermo couple measurement gives the temperature value at one spot. Optical method provides the average temperture which contains spectrum from different areas of high and low temperature. It is likely to misunderstand the observed phenomena to be the non-thermal effects.

For consideration of selective heating, instructive results on the temperature difference evolution were obtained by the numerical simulation of the heat conduction (Yoshikawa and Tokyama, 2009(c)), though the simulation was peformed without coupling the electromagnetic analysis. The ceramic spherical inclusions having large loss factor are embedded in a ceramic matrix having low loss factor. The inclusion particles are placed in the configulation of face centered cubic ( $\mathrm{fcc}$ ) cells, using symmetric boundary, 8 cells are made to be connected, as the schematic illustartion shown in Fig. 18. The same fcc configuration was set as the analysis presented in 3.1. Fig. 19 indicates the temperature evolution for the cases of different particle diameters (This calculation was conducte without using the radiation heat flux boundary condition. All the faces are assumed to be adiabatic.). The larger temperature difference can be evolved in the larger diameter case. This is because the heat generated is propotional to the particle volume but the heat transfered to the surrounding matrix is propotional to the surface area of the particle. This was discussed in 4.2.1. The heat conduction distance (d) can be simply evaluated by

$$
d=\sqrt{2 D \cdot t}
$$

,where $t$ is time and $D$ is the thermal diffusivity $D=\lambda /(\rho C p), \lambda$ : thermal conductivity, $\rho$ : density and Cp: specific heat at constant pressure. Using the material properties of spinel for the matrix and perovskite for the inclusions, $\mathrm{d}$ is estimated to be $100 \mu \mathrm{m}$ for $19 \mathrm{~ms}$. Large temperature difference can be evolved only within several ten milli seconds, if the particles are separated by each other in an order of hundred microns. The simulated temperature distributions of $\mathrm{Cr}_{2} \mathrm{O}_{3}$ /graphite mixture at 300ms is shown in Fig. 20. 

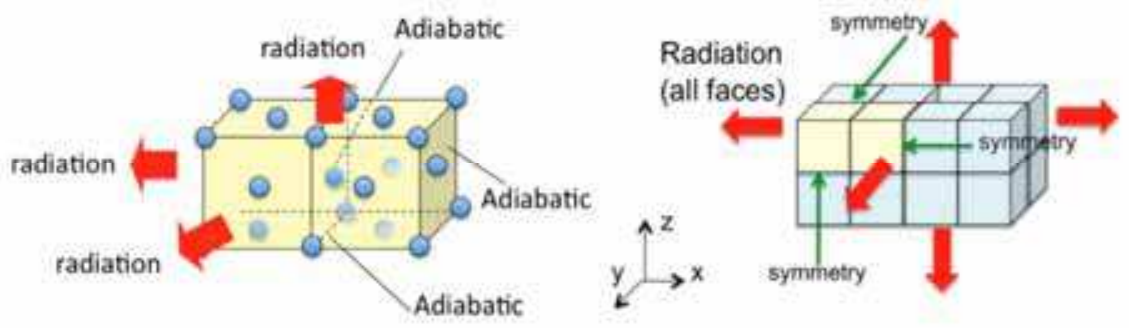

Fig. 18. Calculation region and the boundary conditions.
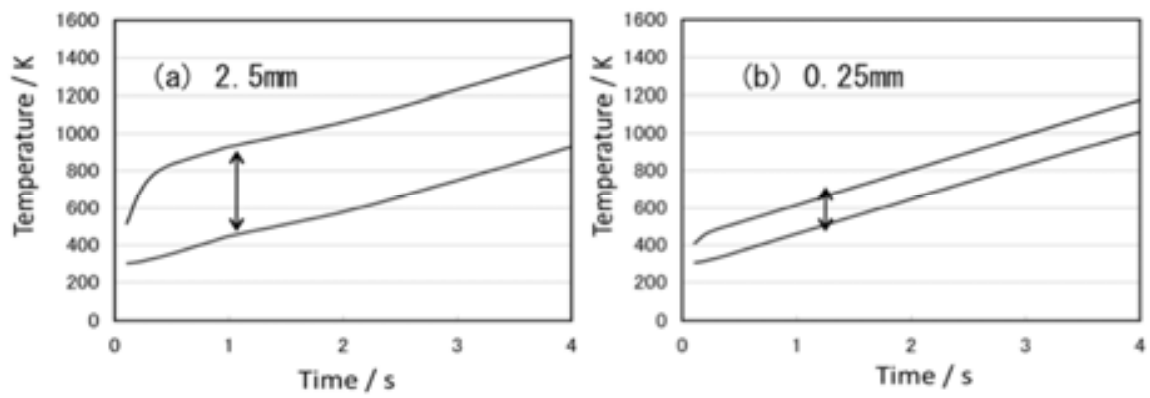

Fig. 19. Temperature difference between the particle center (upper curve) and the matrix minimum (lower curve), for different diameter cases (a): $2.5 \mathrm{~mm}$ and (b) $0.25 \mathrm{~mm}$. Simulations were performed under adiabatic boundary conditions.

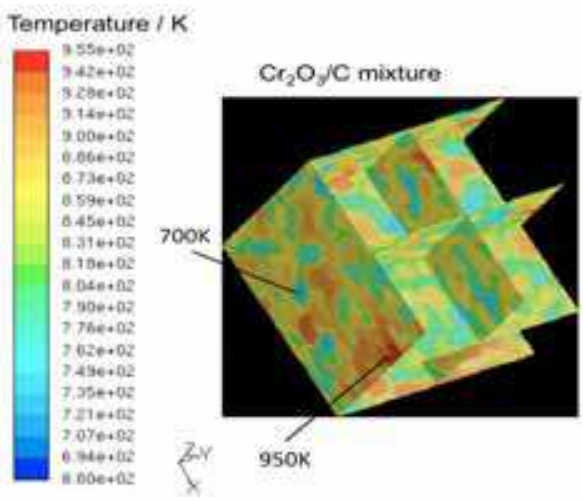

Fig. 20. Temperature distribution in a model cell of powder mixture at $300 \mathrm{~ms}$.

Here, in regards of the ceramic thermal conductivity, important conclusions are that the large temperature difference in ceramics composite or ceramic powder mixture can be evolved only several tens or hundreds seconds. And the temperature difference cannot be evolved in the small scale. Inclusions of millimiter size is required for several hundred degree in ceramics. It is considered that the inhomogeneities in micron scale embedded in ceramics matrix or some crystal imperfectional areas such as the grain boundaries could be different in MW absorbing behavior, however, the temperature difference, if generated, is too small to be detected. 


\subsection{Case 2: The heating accompanied with other phenomena}

Two examples are to be pointed out. One is occurrence of arcing. The arcing or discharge could be generated at large electric field gradient, which is dependent on the material shape. Arcing readily occur at high temperature, because of emission of thermal electrons. Arcing or local plasma could be generated within the small voids, but usually it is difficult to detect them. Moreover, the local arcing or plasma could alter the surface energy and activate the sintering kinetics and some reactions, which are likely to be regarded as the non-thermal effect. The arcing phenomena if happened in the small area, it is not possible to be detected. In our studies, the arcing effect might also be related with the reduction kinetics in $\mathrm{Cr}_{2} \mathrm{O}_{3}$ /graphite mixture wherever the large electric field gradients exist.

The other effect is the generation of high pressure. In heating of liquids, it is reported that super heating occurs. This is simply interpreted that MW internal heating of liquid brings about high temperature area in the internal region of the liquids, however, the difficulty in (homogeneous) nucleation of vapor in the internal region (nucleation boiling) resulted in the temperature rise above the boiling point. This is accompanied by areas having high pressure, comparing with the ambient. So, the observed phenomena, such as increasing the reaction kinetics might be caused by the conditions of high temperature/pressure. The MW digestion processes usually used for dissolving solid particles in the solvents for preparation of ICP analysis specimens are conducted in a closed container and the high pressure/temperature conditions could accelerate the dissolution kinetics. It is still difficult to discuss which effect is dominant to influence the kinetics, either of hydrothermal state or MW irradiation.

\subsection{Case 3: Inherent phenomena raised by MW irradiation}

Atoms or molecules in a gas or liquids, ions in the solid crystals or glass are thermally vibrated. The frequency of thermal vibration is an order of several tens to hundred $\mathrm{THz}$ and is much $\left(10^{4} 10^{5}\right.$ times) higher than that of $\mathrm{MW}$. These thermal vibration frequencies correspond to the "temperature" of the matter. MW is an electromagentic wave, so it is expected the electric or magnetic dipoles in the matter are directly responding to the alternating field, however, it is not necessarily clear how these dipole vibration or rotation excite the thermal vibration of $10^{4}$ times higher frequency. Generally, it is understood that the delay of the rotational motion corresponds to the loss factor and is believed to dissipate and convert to heat, however there no description on these specific processes.

It is of interest to know whether the atomic vibration spectrum excited by MW and the direct excitation by infra-red light (general heating) are different from each other, and whether MW excitation generates the non-Maxwellian distribution of the spectrum. It is expected that the phenomena related with the low frequency vibration spectrum could occur and detected as the non-thermal effects. The magnetic heating mechanism in 3.3 is brought about by resonant electron spin precession motion, inducing the wave number $\mathrm{k}=0$ spin wave is generated and interacts with thermal phonons or creates another phonons. It is possible to interact with the elastic waves which could be observed as the coherent phonon and could give rise the specific effect on the materials' nano structures (Turner, 1960).

As mentioned above, the diference in the local vibration states could be raised in some crystal imperfectional areas such as the grain boundaries could be different in MW absorbing behavior, and effect of nonlinear coupling with the elastic wave was proposed before (Booske, 1992). These phenomena, though not detected as the temperature difference, could induce the non-thermal effect in MW heating. 


\section{Conclusion}

Based on the recent studies by authors' group on the new aspects in MW heating, the essence of the MW heating was discussed. The heating mechanisms were divided into three classes. The magnetic mechanism of which has been recognized but not fully understood with the experimental evidences. This paper provided arguments on contribution of the FMR and discussed its possibility to give rise the non-thermal effect of MW heating.

Separated E- and H- field heating of metal thin film was reported and the application of its heat treatment was introduced. The rapid thermal treatment was proposed taking advantages of the thermal runaway phenomena for the puropse of oxide reduction and glass melting. The non-thermal effect was discussed with possible interpretation of the origins. Some effects to be expected based on the non-Maxwellian distribution of the atomic vibration were suggested important for the future prospects of MW heating applications.

\section{Acknowledgement}

This study was supported by the Grant-in-Aid of Ministry of Education, Sports,Culture, Science and Technology, Japan, Priority Area on Science and Technology of MicrowaveInduced, Thermally Non-Equilibrium Reaction Field (2006-10). Author is grateful to Prof. S. Taniguchi at Tohoku Univ, and Prof. M.Sato at NIFS. The efforts done by the following students and fellows are greatly acknowledged ; Ms.Ishizuka.E, Mr.Mashiko.K, Mr.Sasaki.Y, Mr.Saito.Y, Mr.Kawahira,K, Mr.Kato,T, Dr.Cao,ZP.

\section{References}

Agrawal,D. (2005). in his talk at Japan Institute of Metals (JIM) Symp., Fall Annual Meeting, Hiroshima, Japan.

Bescher, E.P. Sakkar, U. and Mackenzie, J. (1992) „Microwave Processing of Aluminum Silicon Carbide Cermets“, Mat.Res.Symp, Proc., 269 371-378.

Boey,F. Gosling,I. Lye,S.W. (1992). „High-Pressure Microwave Curing Process for an EpoxyMatrix/Glass-Fibre Composite“" , IMater.Process.Technol., 29, 311-319.

Bohren,C and Huffman,D. (1983). "Absorption and Scattering of light by small particles", Wiley and Sons Inc. New York

Booske,J.H. Cooper,R.F and Dobson,I. "Mechanisms for Nonthermal Effects on Ionic Mobility duringMicrowave Processing of Crystalline Solids"(1992) eIMater. Res., 7 [2] 495-501.

Bykov,Y.V. Rybakov,K.I. and Semenov,V.E. (2001). "High-temperature microwave processing of materials", eIPhys. D: Appl. Phys. 34, R55-R57.

Cao,Z.P. Yoshikawa,N and Taniguchi,S. (2009) "Microwave heating behavior of nanocrystalline Au thin films in single-mode cavity", IMater.Res., 24 [1] 268-273.

Cheng,J Roy,R and Grawal,D.A. (2001) “Experimental proof of major role of magnetic field losses in microwave heating of metallic composites" IMater.Sci.Lett. 20 1561-1563.

Cheng,J. Roy,R, and Agrawal,D. (2002). "Radically different effects on materials by separated microwave electric and magnetic fields.", Mater. Res. Innovat., 5 170-177.

Clark,D.E. and Sutton,W.H. (1996). "Microwave processing of materials", Annu. Rev. Mater. Sci., 26 299-331. 
Committee on Microwave Proc. of Mater. (Ed.), (1994). “Microwave processing of materials", An Emerging Industrial. Technology. National Academy Press, Washington D.C. pp.36

Ishizaki,K Nagata,K. (2007) "Selectivity of Microwave Energy Consumption in the Reduction of $\mathrm{Fe}_{3} \mathrm{O}_{4}$ with Carbon Black in Mixed Powder", ISIJ Int. 47 [6] 811-816.

Iwasaki,K. Mashiko,K. Saito,Y. Todoroki,H. Yoshikawa,N. Taniguchi, S. (2009). ISIJInt., 49 [4] 596-601.

Janney.M.A. and Kimrey,H. (1988). "Microwave sintering of alumina at $20 \mathrm{GHz}$, Ceramic Powder Science II, Ceramic Trans. Am. Ceram. Soc., 1 919-924.

Jackson,J.D. (1998), “Classical Electrodynamics”, 3 $3^{\text {rd }}$ ed., Wiley and Sons Inc., new York.

Johnson,P.B. and Christy,R.W., (1972) "Optical Constants of the Noble Metals", Phys. Rev., B6, 4370-4379.

Kato,Y, Shibata,T. and Anbe,T. (2005). "Reaction Mechanism of De-nitration of $\mathrm{UO}_{2}\left(\mathrm{NO}_{3}\right)_{2}$ by the Microwave Heating", epn. eINucl. Sci. Technol. 4 [1] 77-83.

Katz, J.D. (1992). "Microwave Sintering of Ceramics”, Annu. Rev. Mater. Sci., 22 153-170.

Kenkre,V.M. Skala,L. and Weiser,M.W. (1991). "Theory of microwave interactions in ceramic materials: the phenomenon of thermal runaway", J.Mater. Sci., 26 (1991) 2483-2489.

Kingery,W.D.Bowen,H.K. and Uhlmann, D.R., (1975). Introduction to Ceramics, 2nd Ed., John Wiley and Sons, New York, pp. 240.

Kittel,C. (1948). “On the theory of ferromagnetic resonance”, Phys. Rev., 73 155-161.

Kraus,J.D. and Carver,K.R. (1973), “Electromagnetics”,2nd ed. Int. Student Ed. pp.402. McGraw-Hill, New York, USA (For example)

Landau,L.D. and E.M.Lifshitz,E.M. and Pitaevskii,L.P., (1984) "Electrodynamics of Continuous Media",Landau and Lifshitz Course of Theoretical Physis, 2nd ed. Vol. 8 Pergamon Press, Oxford UK.

Leonelli,C. Veronesi,P. Denti,L. Gato.A and Iuliano,L. (2008). “Microwave assisted sintering of green metal parts", J.Mater.Process. Technol. 205 489-496.

Leontovich,L.A. (1948), Investigation of propagation of radiowaves, Pt II.

Lorenson,C. Ball,M.D. Herzig,R and Shaw,H. (1991) „The Microwave Heating Behavior of Metallic-Insulator Composite SYstems“, Mat.Res. Symp. Proc., 189, 279-282.

Ma,J. Diehl, J.F. Johnson,E.J. Martin,K.R. Miskovsky,N.M. Smith,C.T. Weisel.G.J. Weiss,B.L. and Zimmerman,D.T. (2007) "Systematic study of microwave absorption, heating, and microstructure evolution of porous copper powder metal compacts.", JAppl.Phys., 101 074906,1-8.

Mishra,P. Sethi.G and Upadhyaya,A. "Modeling of Microwave. Heating of Particulate Metals", Met. Trans. 37B (2006) 839-845.

Morita,K. Nguyen, V.Q. Nakaoka, R. and Mackenzie, D. (1992), “Immobilization of ash by microwave melting", Mater. Res. Symp. Proc. 269 pp. 471-476.

Oda,S.J. (1992). "Dielectric processing of hazardous materials - prrsent and future opportunities", Mater. Res. Symp. Proc. 269 (1992) pp. 453-464.

Pardavi-Horvath,M., (2000). "Microwave applications of soft ferrites", el Magn. Magn. Mater., 215-216 171-183. 
Polder,D. and Smit,J., (1953). “Resonance Phenomena in Ferrites", Rev. Modern Phys., 25 [1] 89-90.

Rodiger,K. Dreyer,K. Gerdes,T. and Willert-Porada,M. (1998). "Microwave Sintering of Hardmetals.", Int.dRefractoy Met and hard Mater. 16 4-6, 409-416.

Roy,R. Agrawal,D. Cheng.J and Gedevanishvili,S. (1999) “Full sintering of powdered-metal bodies in a microwave field", Nature, 399 668-670.

Roy.R. Peelamedu,R. Hurtt,L. Cheng.J and Agrawal,D. (2002) “Definitive experimental evidence for Microwave Effects: radically new effects of separated E and H fields, such as decrystallization of oxides in seconds", Mater. Res. Innovat., 6 128-140.

Nikawa,Y and Okada,F. (1987). "Selective Heating for Microwave Hyperthermia Using Ferrimagnetic Resonance ", IEEE Trans. Magn. vol. Mag-23,[5] 2431-2433.

Sparks,M. Loudon,R. and Kittel,C. (1961) "Ferromagnetic Relaxation I. Theory of the Relaxation of the Uniform Precession and the Degenerate Spectrum in Insulatord at Low Temperatures", Phys. Rev., 122 [3] 791-803.

Sparks,M. (1964). "Ferromagnetic Relaxation Theory”, McGraw-Hill, New York, USA

Turner.E (1960). "Interaction of Phonons and Spin Waves in Yttrium Iron Garnet", Phys. Rev. Lett., 5 [3] 100-101.

URL: http://www.gallawa.com/microtech/history.html (browsed in Aug. 2010)

Walkievicz,J. Kazonich,G. and MacGill,S.L. (1988). “Microwave Heating Characteristics of Minerals and Compounds", Min. Metall. Processing $539-42$.

Walton,D. Snape,S. Rolph,T.C. Shaw.J and Share,J. (1996). "Application of ferrimagnetic resonance heating to palaeointensity determinations", Phys. Earth Planet.Inter., 94 (1996) 183-186.

Wang, Z.J. Cao,Z.P Ohtsuka,Y. Yoshikawa,N Kokawa,H and Taniguchi,S. (2008) “Low temperature growth of ferroelectric lead zirconium titanate thin films using the magnetic field of low power 2.45GHz microwave irradiation" Appl. Phys. Lett. 92 222905-

Whittaker,A.G. and Mingos, D.P. (1995). “Microwave-assisted solid-state reactions involving metal powders", J. Chem. Soc., Dalton Trans. 2073-2079.

Wroe,R and Rowley,A.T. (1996) "Evidence for a Non-Thermal. Microwave. Effect in the Sintering of Partially. Stabilized Zirconia", J.Mater.Sci., 31 2019-2026.

Yoshikawa,N. Ishizuka,E and Taniguchi,S. (2006). "Heating of Metal Particles in a SingleMode Microwave Applicator." Mater. Trans., 47 [3] 898-902.

Yoshikawa,N Ishizuka,E Mashiko,K. Taniguchi,S. (2007). “Carbon Reduction Kinetics of $\mathrm{NiO}$ by Microwave Heating of the Separated Electric and Magnetic Fields.", Metal. Mater. Trans. B,38 [6],863-868.

Yoshikawa,N. Wang,H.C. Mashiko,K and Taniguchi,S. (2008a). "Microwave heating of Soda-Lime Glass by Addition of Iron Powder." el Mater. Res., 23 [6] 1564-1569.

Yoshikawa,N. Mashiko,K. Sasaki,Y. Taniguchi,S. and Todoroki,H. (2008b). “Microwave Carbo-thermal Reduction for Recycling of $\mathrm{Cr}$ from Cr-containing Steel Making Wastes." ISIJInt. 48 [5] 697-702.

Yoshikawa,N. (2009a). "', Fundamentals and Application of Mcrowave heating of Metals", Materia, elopan (Bulletin of stpan Inst.Metals), 48 [1] 3-10. 
Yoshikawa,N. Wang,H.C. and Taniguchi,S. (2009b) “Application of Microwave Heating to Reaction between Soda-Lime Glass and Liquid Al for Fabrication of Composite Materials" Mater. Trans. 50 [5] 1174-1178.

Yoshikawa,N. Tokuyama,Y. (2009b). "Numerical Simulation of Temperature Distribution in Multi-Phase Materials as a Result of Selective Heating by Microwave Energy", $d$ Microwave Power and Electromagnetic Energy, 43 [1], 27-33.

Yoshikawa,N. and Kato,T. (2010) “Ferromagnetic Resonance Heating of Fe and $\mathrm{Fe}_{3} \mathrm{O}_{4}$ by 5.8 GHz Microwave Irradiation”, J.Phys.D: Appl. Phys. 43425403. 


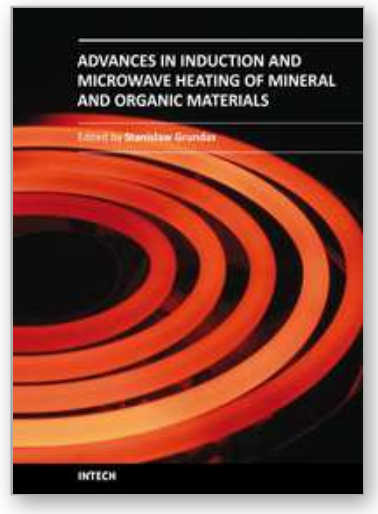

\author{
Advances in Induction and Microwave Heating of Mineral and \\ Organic Materials
}

Edited by Prof. StanisÅ,aw Grundas

ISBN 978-953-307-522-8

Hard cover, 752 pages

Publisher InTech

Published online 14, February, 2011

Published in print edition February, 2011

The book offers comprehensive coverage of the broad range of scientific knowledge in the fields of advances in induction and microwave heating of mineral and organic materials. Beginning with industry application in many areas of practical application to mineral materials and ending with raw materials of agriculture origin the authors, specialists in different scientific area, present their results in the two sections: Section 1-Induction and Microwave Heating of Mineral Materials, and Section 2-Microwave Heating of Organic Materials.

\title{
How to reference
}

In order to correctly reference this scholarly work, feel free to copy and paste the following:

Noboru Yoshikawa (2011). Recent Studies on Fundamentals and Application of Microwave Processing of Materials, Advances in Induction and Microwave Heating of Mineral and Organic Materials, Prof. StanisÅ,aw Grundas (Ed.), ISBN: 978-953-307-522-8, InTech, Available from:

http://www.intechopen.com/books/advances-in-induction-and-microwave-heating-of-mineral-and-organicmaterials/recent-studies-on-fundamentals-and-application-of-microwave-processing-of-materials

\section{INTECH}

open science | open minds

\section{InTech Europe}

University Campus STeP Ri

Slavka Krautzeka 83/A

51000 Rijeka, Croatia

Phone: +385 (51) 770447

Fax: +385 (51) 686166

www.intechopen.com

\section{InTech China}

Unit 405, Office Block, Hotel Equatorial Shanghai

No.65, Yan An Road (West), Shanghai, 200040, China

中国上海市延安西路65号上海国际贵都大饭店办公楼 405 单元

Phone: +86-21-62489820

Fax: +86-21-62489821 
(C) 2011 The Author(s). Licensee IntechOpen. This chapter is distributed under the terms of the Creative Commons Attribution-NonCommercialShareAlike-3.0 License, which permits use, distribution and reproduction for non-commercial purposes, provided the original is properly cited and derivative works building on this content are distributed under the same license. 